REVIEW ARTICLE

\author{
V. Cuvinciuc \\ A. Viguier \\ L. Calviere \\ N. Raposo \\ V. Larrue \\ C. Cognard
}

F. Bonneville

\section{Isolated Acute Nontraumatic Cortical Subarachnoid Hemorrhage}

SUMMARY: Our aim was to review the etiologic background of isolated acute nontraumatic cSAH. While SAH located in the basal cisterns originates from a ruptured aneurysm in approximately $85 \%$ of cases, a broad spectrum of vascular and even nonvascular pathologies can cause acute nontraumatic $\mathrm{SAH}$ along the convexity. Arteriovenous malformations or fistulas, cortical venous and/or dural sinus thrombosis, and distal and proximal arteriopathies (RCVS, vasculitides, mycotic aneurysms, Moyamoya, or severe atherosclerotic carotid disease) should be sought by noninvasive imaging methods or/and conventional angiography. Additionally, PRES may also be a source of acute cSAH. In elderly patients, cSAH might be attributed to CAA if numerous hemorrhages are demonstrated by GRE T2 images. Finally, cSAH is rarely observed in nonvascular disorders, such as abscess and primitive or secondary brain tumors.

\begin{abstract}
ABBREVIATIONS: CAA = cerebral amyloid angiopathy; $\mathrm{CSAH}=$ cortical subarachnoid hemorrhage; CTA = CT angiography; CTV = CT venography; CVT = cerebral venous thrombosis; DSA = digital subtraction angiography; DWI = diffusion-weighted imaging; FLAIR = fluid-attenuated inversion recovery; Gd = gadolinium; GRE T2 = gradient echo T2-weighted imaging; MRA = MR angiography; MRV = MR venography; PRES = posterior reversible encephalopathy syndrome; RCVS = reversible cerebral vasoconstriction syndrome; SAH = subarachnoid hemorrhage; SWI = susceptibility-weighted imaging; TIA = transient ischemic attack; TOF = time of flight
\end{abstract}

$\mathbf{N}$ ontraumatic (spontaneous) SAH arises in approximately $85 \%$ of cases from rupture of a saccular aneurysm at the base of the brain. Nonaneurysmal perimesencephalic hemorrhages account for another $10 \% .{ }^{1}$ Arterial dissection and pituitary apoplexy are additional causes of SAHs that are centered in the basal cisterns.

Spontaneous acute cSAH observed at the convexity of the brain is a relatively rare entity, seldom described, ${ }^{2-4}$ with various vascular and nonvascular causes, including the following: CVT (both dural and cortical), ${ }^{5,6}$ vascular malformations (pial arteriovenous malformations, dural arteriovenous fistulas, and cavernomas), RCVS, ${ }^{7}$ vasculitides, ${ }^{8,9}$ infectious aneurysms, ${ }^{10}$ Moyamoya disease or syndrome, ${ }^{11}$ severe atherosclerotic carotid disease, PRES, ${ }^{12} \mathrm{CAA},{ }^{13}$ and nonvascular disorders, such as primary and secondary brain neoplasms ${ }^{14-16}$ or even abscess (Table). ${ }^{17}$

The purposes of this work were the following: first to propose a diagnostic work-up that covers the wide spectrum of potential underlying causes of $\mathrm{cSAH}$ and then to review the specific findings of each etiology that may be responsible for cSAH.

From the Department of Neuroradiology (V.C., C.C., F.B.), University Hospital, Toulouse, France; and Department of Vascular Neurology (A.V., L.C., N.R., V.L.), Rangueil University Hospital, Toulouse, France.

Previously presented as a poster at: Annual Meeting of the American Society of Neuroradiology and Neuroradiology Education and Research Foundation, May 16-21, 2009; Vancouver, British Columbia, Canada. It received a "Cum Laude" award.

Please address correspondence to Fabrice Bonneville, MD, Department of Neuroradiology, Hôpital Rangueil, 1, Av du Professeur Jean Pouhlès, 31059 Toulouse, France; e-mail: bonneville.f@chu-toulouse.fr

Indicates open access to non-subscribers at www.ajnr.org

DOI 10.3174/ajnr.A1986

\begin{tabular}{l}
\hline Etiology of cSAH \\
\hline Etiology \\
\hline Pial arteriovenous malformations \\
Dural arteriovenous fistulas \\
Arterial dissection \\
Dural/cortical venous thrombosis \\
Vasculitides \\
RCVS \\
PRES \\
High-grade stenosis \\
Endocarditis \\
CAA \\
Coagulation disorders \\
Abscess \\
Cavernoma \\
Primary and secondary brain tumors \\
\hline
\end{tabular}

\section{Imaging Protocol}

The clinical presentation of cSAH might be different from the classic thunderclap headache reported in aneurysmal SAH; cSAH presents with focal deficits, especially in older patients, which might suggest a TIA, migraine with aura, or epileptic seizures. ${ }^{18}$ The thunderclap headache is mostly seen in younger patients and is more specific for CVT or RCVS.

Patients with such symptoms should undergo emergent nonenhanced CT. Cortical SAH, if present, is then noticed as a slight sulcal hyperattenuation. The presence of a spontaneous cSAH should prompt dedicated brain CTA, with a dual passage at both arterial and venous phases. This double acquisition is efficient in detecting aneurysms, vascular malformations, and CVT and offers valuable information with respect to high-grade stenosis, Moyamoya, RCVS, and vasculitides.

However, one of the major issues of $\mathrm{CSAH}$ is its detection on neuroimaging studies. Nonenhanced head CT has a sensitivity of $>90 \%$ in the acute stage of $\mathrm{SAH}$, but the sensitivity falls off rapidly, especially if minimal hemorrhage is present. ${ }^{19}$ 
Thus, cSAH may be barely visible on nonenhanced CT, and its presence should be confirmed by MR imaging. If needed for the subsequent work-up, to cover the wide spectrum of entities potentially responsible for $\mathrm{CSAH}, \mathrm{MR}$ imaging should include FLAIR, GRE T2 sequences, DWI, 3D TOF MRA, contrast-enhanced venography, and pre- and postgadolinium T1weighted imaging.

FLAIR is known to be extremely sensitive to pathologies that affect the subarachnoid space ${ }^{20-22}$ and is specifically superior to $\mathrm{CT}$ in depicting the acute and subacute low-grade $\mathrm{SAH},{ }^{23}$ as is often the case with cSAH. However, subarachnoid FLAIR hyperintensity is not specific for SAH and has been described in various other conditions, such as meningitis, ${ }^{24}$ leptomeningeal metastasis, ${ }^{25}$ leptomeningeal melanosis, ${ }^{21}$ status epilepticus, ${ }^{26}$ supplemental $\mathrm{O}_{2}$ administration, ${ }^{27}$ intravenous anesthetic agents, ${ }^{28,29}$ previous intravenous iodinated or gadolinium contrast material, ${ }^{30,31}$ residual oil-based contrast medium, ${ }^{21}$ and artifacts. ${ }^{20,21}$ In addition to confirming the cSAH, the FLAIR sequence, together with DWI, is essential for demonstrating cerebral parenchymal signal-intensity abnormalities found in several conditions that may lead to $\mathrm{cSAH}$, as detailed above.

GRE T2 is sensitive to the magnetic susceptibility effect of decomposition products of hemoglobin. However, GRE T2 is less sensitive than FLAIR for the detection of acute $\mathrm{SAH} .{ }^{23}$ In this setting, GRE T2 is extremely useful because it may confirm the hemorrhagic nature of the sulcal abnormality, detect previous intraparenchymal or cortical hemorrhages, and finally evaluate the possible presence of cerebral cortical or dural venous thrombosis. ${ }^{32-34}$

SWI, which is a relatively new MR imaging sequence based on susceptibility differences between tissues, is extremely sensitive to paramagnetic substances and venous blood vessels. ${ }^{35}$ This sequence has been shown to be far superior to conventional T2 or GRE T2 sequences in detecting microbleeds and cortical hemosiderosis $^{36,37}$ and could thus be of potential value in cases of $\mathrm{CSAH}$.

Finally, despite the efficacy of this noninvasive protocol, DSA remains valuable in cases of cSAH, when diagnosis is unclear or unconfirmed, or as a tool for direct therapeutic interventions (Fig 1).

\section{Etiologies of CSAH}

\section{CVT}

CVT (Figs 2 and 3) is a relatively uncommon but probably underestimated disorder for which the imaging diagnosis is crucial because of its varied clinical manifestations. It usually affects young adults, mostly women, ${ }^{38}$ who present with generalized or focal neurologic symptoms and/or signs of intracranial hypertension. At imaging, half of the patients have parenchymal abnormalities (cortical/subcortical edema with or without hemorrhage), but $\mathrm{SAH}$ is increasingly recognized as a presentation, both for cortical and dural sinus thrombosis., The exact mechanism of SAH associated with CVT is not known but may result from venous hypertension and subsequent rupture of dilated thin-walled subarachnoid cortical veins. ${ }^{5}$ The topography of the SAH is variable, but it usually spares the basal cisterns. ${ }^{6}$

\section{$\mathrm{cSAH}$}

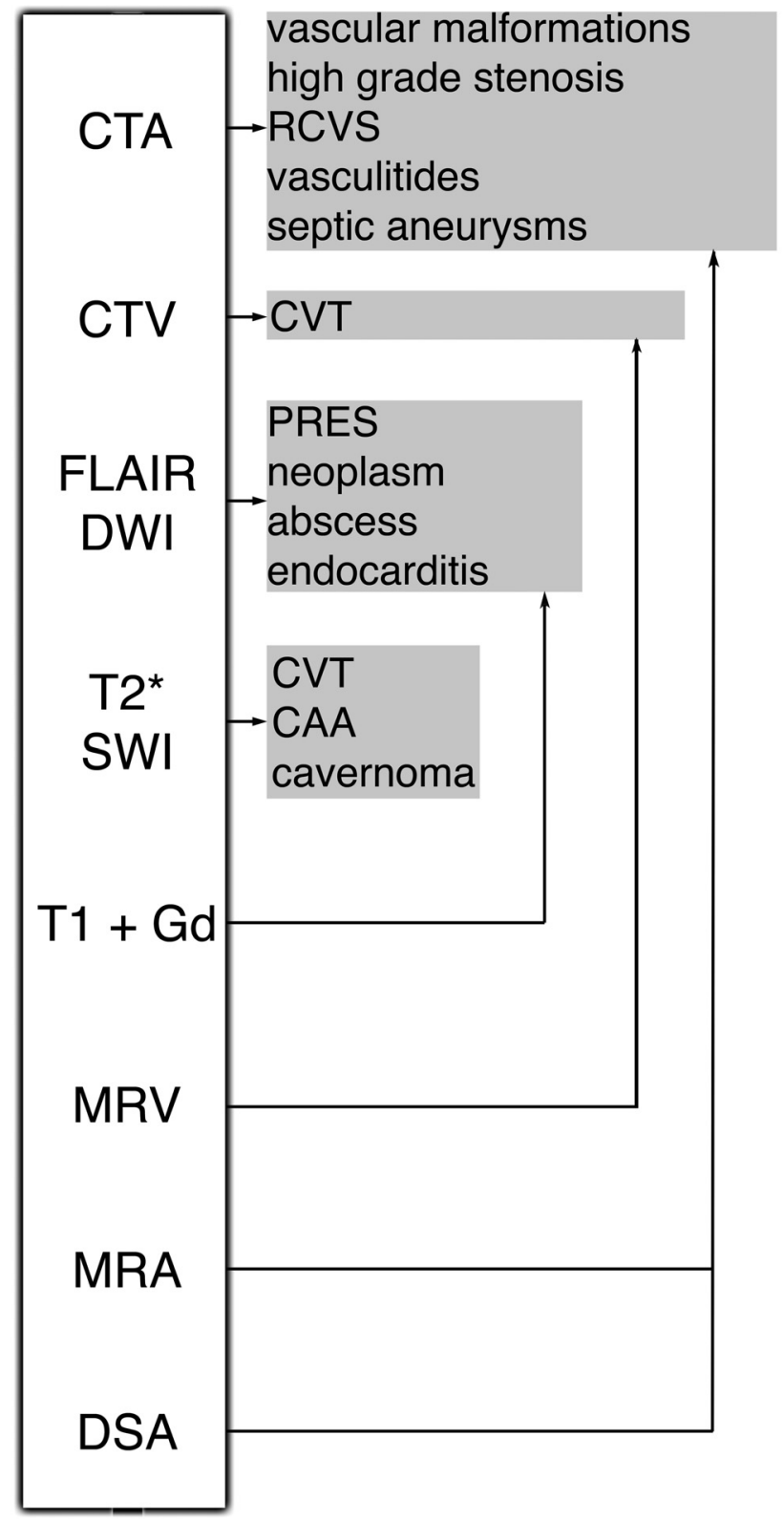

Fig 1. Imaging algorithm and main causes of $\mathrm{CSAH}$.

For diagnosis of CVT, multidetector CT venography provides excellent sensitivity and specificity for the depiction of dural sinus thrombosis, but its value remains to be proved for isolated cortical venous thrombosis. ${ }^{39}$

At MR imaging, the GRE T2 sequence appears to be one of the most useful MR images for the diagnosis of dural and cortical CVT because it directly shows the thrombus with an abnormal low signal intensity at the acute phase due to the magnetic susceptibility effect of deoxyhemoglobin. ${ }^{34}$ This is particularly important in the acute setting, when T1 and T2 spin-echo sequences demonstrate almost no signal-intensity abnormalities. In the subacute phase, the thrombus is hyperintense on T1- and T2-weighted images due to methemoglobin. MR venography, especially gadolinium-enhanced techniques, allows a confident diagnosis. ${ }^{6,40}$ 

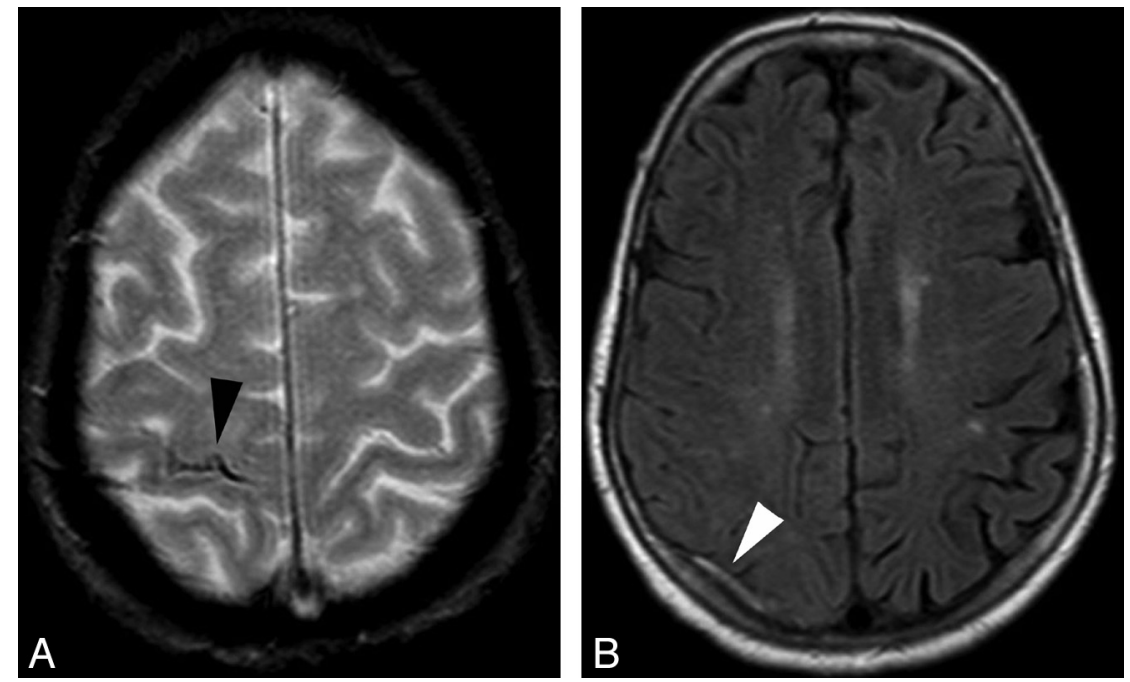

Fig 2. Cortical venous thrombosis. A, Axial GRE T2 image shows right central sulcus SAH (black arrowhead). B, Axial FLAIR image shows a thrombosed right cortical parietal vein (white arrowhead).
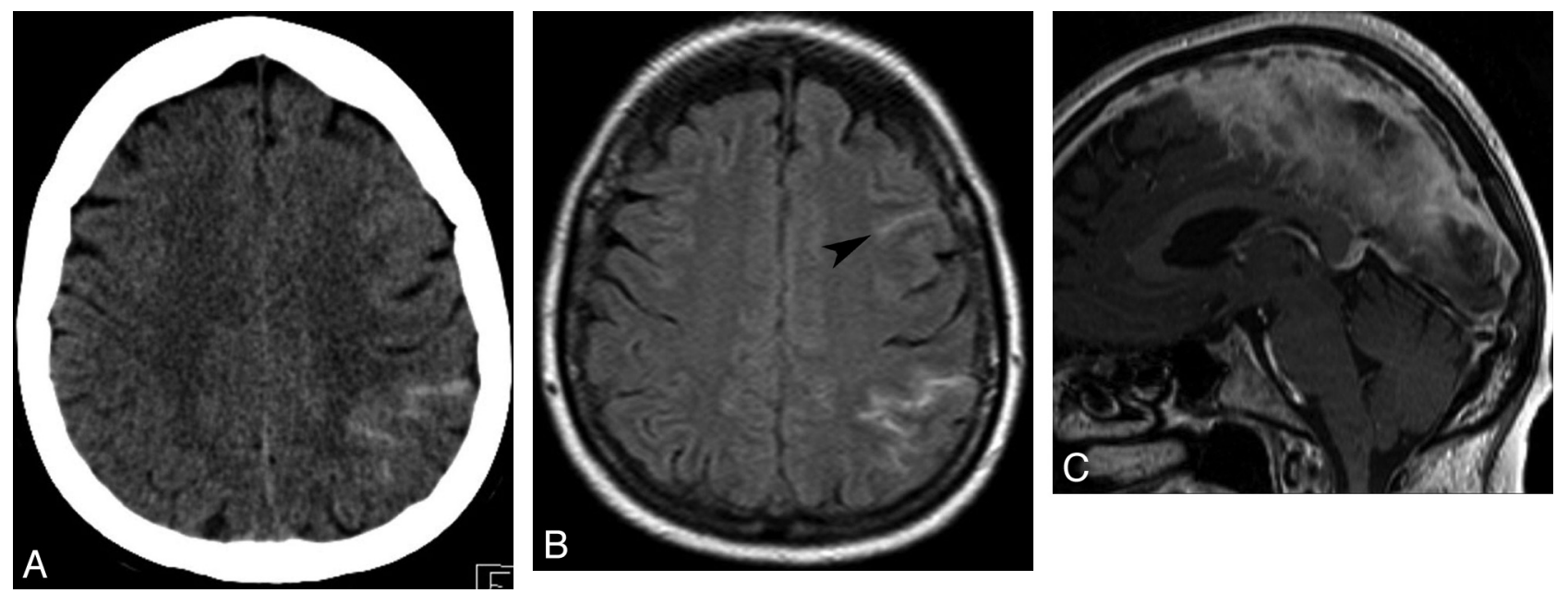

Fig 3. Dural sinus thrombosis. A, Axial brain CT shows left peri-rolandic SAH. B, Axial FLAIR image confirms the sulcal hemorrhage, which is also visible more anteriorly (arrowhead). $C$, Sagittal contrast-enhanced $\mathrm{T} 1$ shows a filling defect in the superior sagittal sinus.

\section{Vascular Malformations}

Although vascular malformations are classic causes of hemorrhagic stroke, pial and dural arteriovenous malformations typically present with parenchymal hematoma or basal SAH and only rarely with isolated cSAH. The presence of vascular malformations is suspected on CTA and usually confirmed on cerebral DSA.

Similarly, cavernomas are rarely described as a cause of SAH. ${ }^{41}$ However, superficial and leptomeningeal cavernomas may be the source of cSAH. Extra-axial cavernomas are different from their intra-axial counterparts, being more vascular with a homogeneous contrast enhancement, showing signs of chronic or acute hemorrhage, and having a tendency to bleed during surgery. ${ }^{41}$

\section{RCVS}

RCVS (Fig 4) is a new name unifying a spectrum of separately described overlapping pathologies that have some fundamental clinical and imaging features in common: severe headache, variable neurologic deficits, and reversible arterial abnormalities (dilations and stenoses). ${ }^{7,42,43}$
The pattern of presentation is multiple thunderclap headaches, either spontaneous or secondary to postpartum or various vasoactive substances (cannabis, selective serotonin-recapture inhibitors, and nasal decongestants). ${ }^{7}$ The pathologic mechanism might be related to a transient disturbance of cerebral autoregulation. ${ }^{44}$

In addition to the multiple intracranial arterial focal stenoses first depicted by noninvasive angiography and eventually confirmed by DSA, neuroimaging may show parenchymal ischemic or hemorrhagic lesions and also isolated cSAH. Vascular evaluation is performed by CTA, MRA, transcranial Doppler sonography, or DSA. Diagnosis is subsequently confirmed by the reversibility of the arterial lesions within 3 months.

\section{Vasculitides}

It is beyond the scope of this article to discuss in depth the wide variety of primary or secondary cerebral vasculitides. This highly heterogeneous group of disorders might be complicated by cerebral hemorrhage (including isolated cSAH) due to inflammation of the wall of the blood vessels. ${ }^{45}$ 

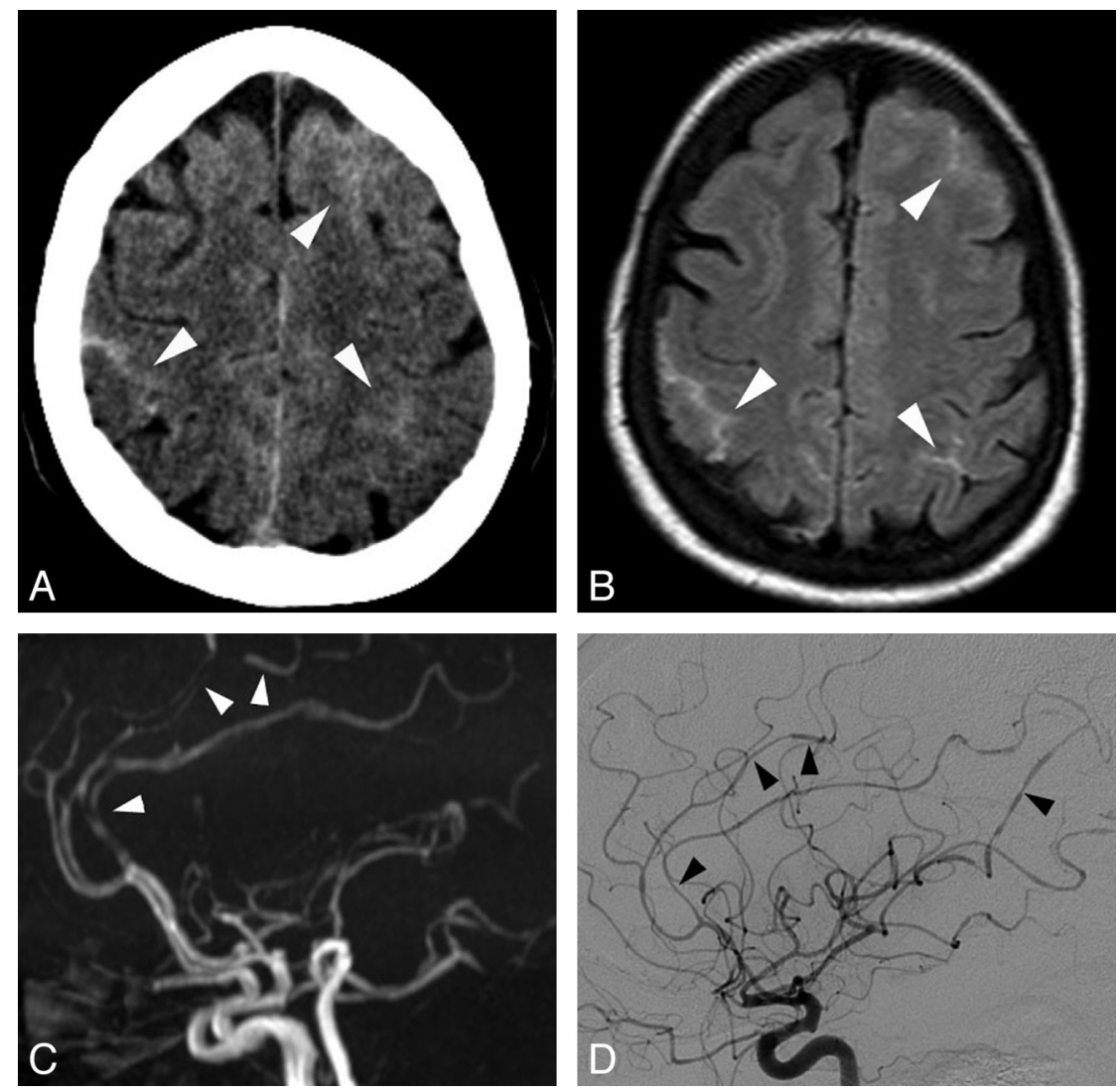

Fig 4. RCVS. A, Axial brain CT scan shows bilateral frontoparietal sulcal SAH (white arrowheads). B, Axial FLAIR image confirms the cSAH (white arrowheads). C, Lateral projection of 3D TOF MRA shows multiple arterial stenoses and dilations, mainly on the anterior cerebral artery branches (white arrowheads). D, Lateral projection of a right internal carotid angiogram shows multiple stenoses and dilations on both anterior cerebral and middle cerebral arteries (black arrowheads). Note that DSA, even if it showed more clearly the arterial abnormalities, did not change the previously suspected diagnosis. Follow-up MRA performed at 3 months demonstrated disappearance of the arterial lesions (not shown).
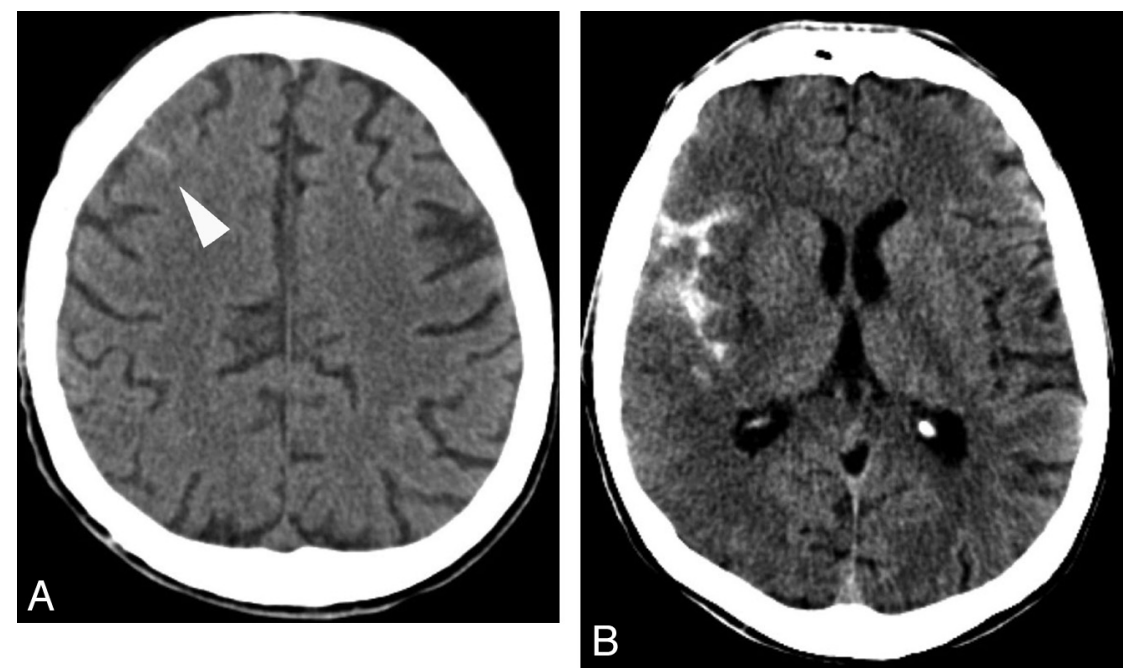

Fig 5. Endocarditis. A, Axial brain CT scan shows an isolated slight right frontal subarachnoid hyperattenuation. B, Because of clinical aggravation the next day, another brain CT was performed and demonstrated a larger right Sylvian SAH. Subsequent MR imaging showed a right middle cerebral artery infarction due to M1 occlusion (not shown), which precluded the exact identification of this SAH origin.

Primary angiitis of the central nervous system is the main differential diagnosis of RCVS at vascular imaging. However, it is suspected in the clinical context of a subacute onset of symptoms, with nonreversible small and medium-sized arterial stenoses and dilations and is confirmed by histologic examination. ${ }^{45}$

\section{Infectious Origin}

Cortical SAH may be seen after rupture of infectious aneurysms (caused by infective endocarditis, meningitis, or rarely cryptogenically) (Fig 5). ${ }^{10}$ Headache is diffuse and vague, rather than the typical excruciating headache of noninfectious aneurysmal SAH. Usually, there are also general symptoms (eg, anorexia, weight 

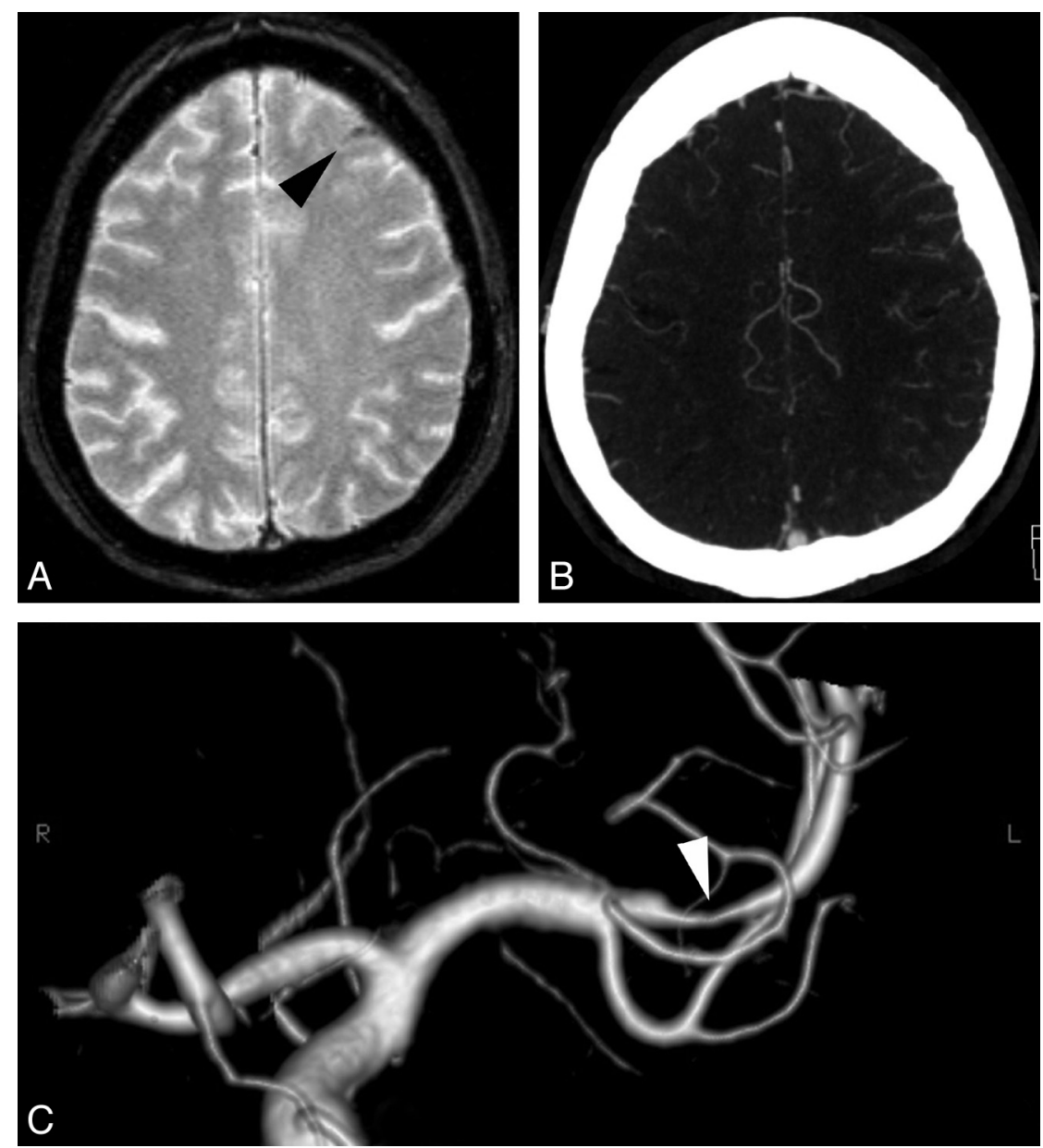

Fig 6. Pial vasodilation. $A$, Axial GRE T2 image shows a left frontal sulcal SAH (black arrowhead), possibly located in the "watershed" territory between the anterior and the middle cerebral arteries. B, Axial maximum-intensity-projection reconstruction of CTA shows an asymmetry of the distal arteries, in favor of left pial vasodilation. $C$, Frontal projection of $3 \mathrm{D}$ angiography of the left carotid artery reveals a severe stenosis at the origin of the M2 branch (white arrowhead).

loss, malaise, or fever). ${ }^{46}$ In infective endocarditis, MR imaging might show associated lesions (ischemic, microbleeds, brain abscesses). ${ }^{47}$ DSA might be used for the diagnosis of distal arterial lesions and for therapeutic purposes.

Sometimes there is no evidence of aneurysm in patients with cSAH and infectious endocarditis. The mechanism is not very well understood, but it might be related to septic embolic infarction, ${ }^{48}$ rupture of a venous or capillary vessel, rupture of an occult aneurysm, or focal arteritis. ${ }^{46}$

Besides endocarditis, other infections might be complicated by SAH because of vasculitis with or without aneurysmal lesions, such as human immunodeficiency virus, ${ }^{49}$ Borrelia burgdorferi, ${ }^{50}$ aspergillosis, ${ }^{51}$ and varicella zoster ${ }^{9}$; but the topography of the hemorrhage tends to be basal.

Rarely, an abscess might present with cSAH, due probably to focal arteritis of adjacent cortical vessels. ${ }^{17}$

\section{Moyamoya}

Moyamoya is an angiographic finding, characterized by deep and superficial abnormal vascular networks triggered by progressive stenosis or occlusion of the supraclinoid carotid artery. ${ }^{52}$ According to a recent classification, patients with wellrecognized associated conditions (eg, sickle cell disease, neurofibromatosis type 1 , cranial therapeutic irradiation, and Down syndrome) are considered as having a Moyamoya syn- drome, while patients with no known associated risk factors are said to have Moyamoya disease. ${ }^{53}$

Most intracranial hemorrhages in Moyamoya occur within the brain parenchyma or the ventricles. SAH is unusual and may result from the rupture of associated saccular aneurysms with basal topography. ${ }^{11,52}$ However, there are reports of cSAH observed exclusively at the cerebral convexity, which is thought to be caused by the rupture of dilated fragile cortical arterioles that are stressed because they supply the watershed areas of hypoperfused carotid territories. ${ }^{11,52}$

\section{High-Grade Stenosis}

Similar to the above-described mechanism of cSAH observed in Moyamoya, cSAH might occur in high-grade atherosclerotic stenosis of intra- or extracranial cerebral arteries by the rupture of dilated fragile compensatory pial vessels (Fig 6). The cSAH is located at the borderline between the dilated territory and the normally perfused one in these cases. Arterial imaging shows the stenosis at the carotid bifurcations, carotid siphons, or intracranial arteries, without the typical "puff of smoke" appearance of lenticulostriate arteries characteristic of Moyamoya. Additionally, dilated collaterals might be seen, either pial (between the internal carotid and vertebrobasilar circulation) or transdural (between cerebral and dural circulation). 

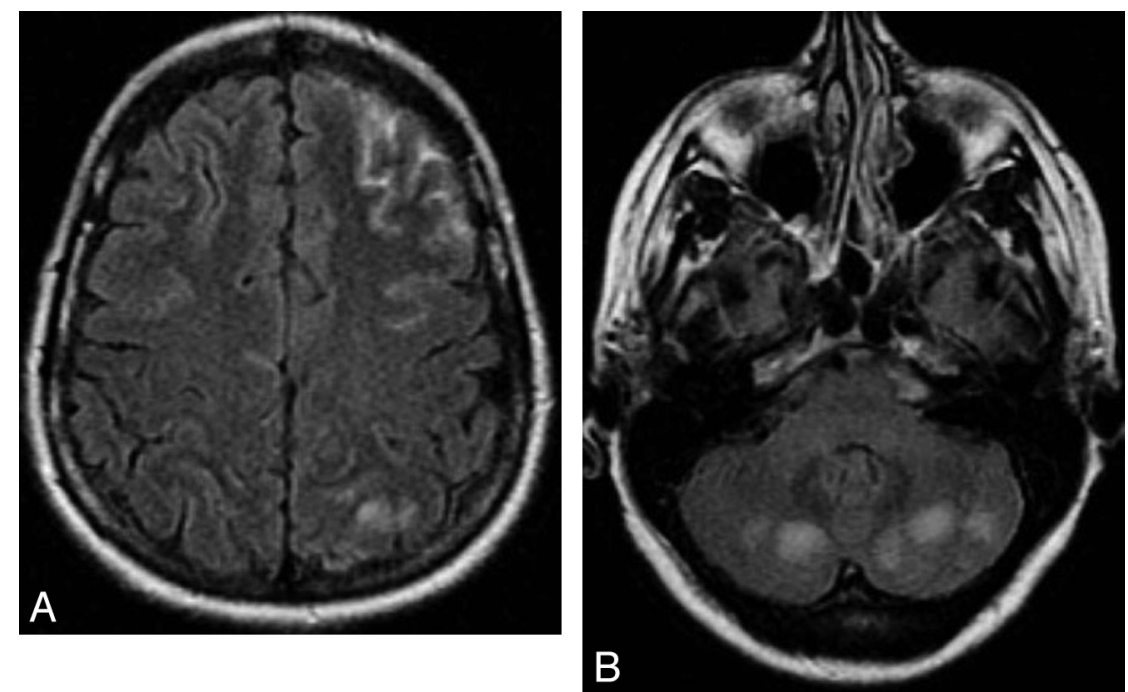

Fig 7. Eclampsia-related PRES. A, Axial FLAIR image shows a left frontal SAH and a left parietal subcortical hyperintensity. B, Axial FLAIR image shows bilateral cerebellar hyperintensities, very suggestive of PRES lesions.

\section{PRES}

PRES (Fig 7) is a clinical and neuroradiologic entity of various origins, characterized by a variety of symptoms, including headache, altered mental status, visual loss, seizures, and loss of consciousness. ${ }^{54-57} \mathrm{CT}$ and MR imaging typically demonstrate bilateral predominantly posterior cerebral edema, which is usually shown on DWI to be of vasogenic origin. ${ }^{55,56}$ When present, the cytotoxic edema is reported to be associated with an adverse outcome. ${ }^{58}$

The topography of these parenchymal lesions resembles the brain watershed regions, with a variable involvement of cortical or subcortical structures and of deep gray and white matter.

Hemorrhage in PRES is described in 5\%-17\% of patients, ${ }^{57,59}$ with 3 different almost equally distributed patterns: parenchymal hematoma, minute hemorrhages $(<5 \mathrm{~mm})$, and cSAH. The mechanism is unclear, probably due to reperfusion lesions or rupture of pial vessels secondary to severe hypertension and impaired cerebral autoregulation. ${ }^{60}$

\section{CAA}

CAA (Fig 8) is a degenerative angiopathy, characterized by deposition of the beta-amyloid peptide in the wall of medium and small cortical and meningeal arteries. It is probably an underdiagnosed disease because it can be asymptomatic. When symptomatic, the clinical presentation varies from a sudden neurologic deficit (related to an intracerebral hematoma) to seizures, transient symptoms, and progressive dementia. According to the Boston criteria, the diagnosis is suspected in elderly patients with histopathologic or MR imaging evidence of multiple lobar, cortical, or subcortical hemorrhages. $^{61}$

CAA is seldom described as a cause of symptomatic or occult $\mathrm{SAH}^{13,62}$ but is increasingly recognized as a cause of superficial hemosiderosis. ${ }^{13,36,63}$ The clinical presentation of these patients with $\mathrm{CSAH}$ is distinct because headache is usually absent. Rather, patients have transient focal neurologic deficits, mimicking a TIA; focal seizures; or migraine aura-like symptoms. Unenhanced head CT shows a slight, sometimes barely visible, sulcal hyperattenuation, most frequently depicted in the perirolandic region. This discrete abnormality must be sought specifically by radiologists because it is usually not flagrant and also because it is not the usual abnormality expected on an emergency head CT in case of TIA. Subsequent MR imaging confirms the subarachnoid bleeding as a hyperintense signal intensity on FLAIR. Additionally, with GRE T2 and notably SWI, MR imaging shows multiple lobar subcortical hemorrhagic sequelae (macrohemorrhages or microbleeds), classically sparing the deep gray matter, and superficial hemosiderosis. This superficial hemosiderosis has a supratentorial distribution, often extending beyond the site of the acute bleeding (as initially seen on FLAIR), suggesting that previous occult cSAH has occurred. Patients with cSAH and suspicion of CAA should be followed carefully because subcortical hematoma has reportedly occurred within a few days in these patients. ${ }^{64,65}$

The exact origin of cSAH in CAA remains unknown, but rupture of leptomeningeal arteries weakened by amyloid deposits seems a plausible hypothesis. ${ }^{66}$

\section{Primary Brain Tumors}

Although uncommon, there are reports ${ }^{14-16}$ of brain tumors presenting with SAH. Sometimes, the tumor is not obvious on the emergency nonenhanced head CT, but the sulcal topography of the SAH indicates an MR imaging examination rather than DSA.

\section{Secondary Brain Tumors}

Leptomeningeal metastasis is the main differential diagnosis of isolated cSAH, due to the close, even similar, CT and FLAIR findings. Clinical presentation with sudden excruciating headache favors the possibility of SAH, but often it does not occur in cases of cortical SAH. The presence of a primary tumor is not helpful, because in this oncologic context, $\mathrm{CSAH}$ may be due to coagulopathies, cerebral vein thrombosis, PRES, or RCVS (associated with chemotherapy). ${ }^{7,56,67}$ Rapidly progressing multiple cranial nerve paresis is more specific for leptomeningeal metastasis. 

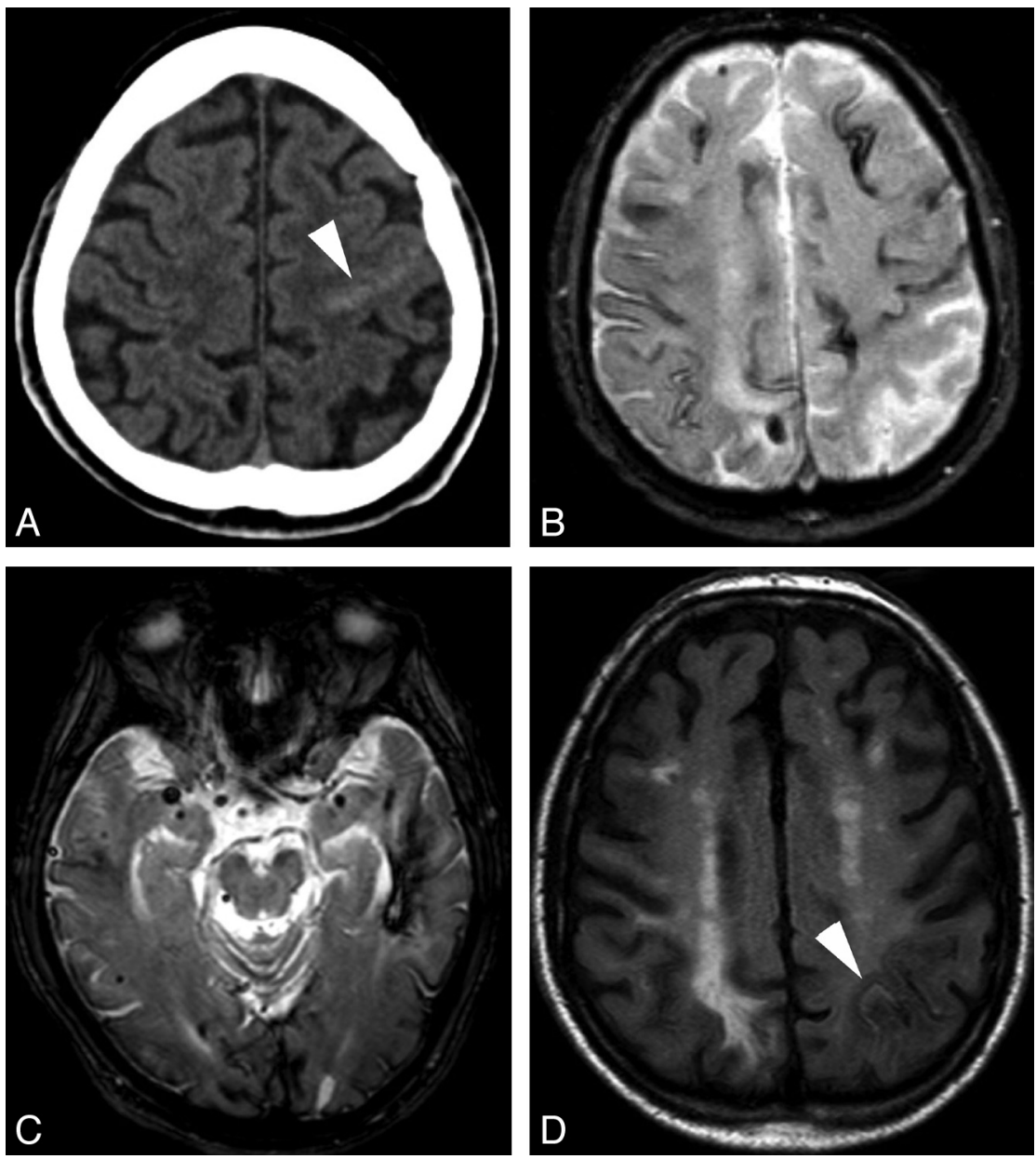

Fig 8. CAA. $A$, Axial brain CT scan shows a subtle left rolandic hyperattenuation favoring minimal SAH (white arrowhead). $B$ and $C$, Axial GRE T2 images show left temporal lobar hemorrhage, multiple microbleeds, and cortical hemosiderosis. D, Axial FLAIR image obtained after 7 months shows a new asymptomatic SAH in a left parietal sulcus (white arrowhead), which was previously normal (see $B$ ).

Hypointensity on GRE T2 confirms a hemorrhagic component, but acute SAH is hyperintense on GRE T2. Leptomeningeal enhancement on contrast-enhanced T1 or FLAIR favors metastases. Gadolinium-enhanced $\mathrm{T} 1$ with fat saturation and section thickness thinner than $3 \mathrm{~mm}$ should be acquired in the posterior fossa to identify cranial nerve involvement if there is a suspicion of leptomeningeal metastasis.

\section{Conclusions}

Isolated nontraumatic cSAH is a subtle finding on emergent head CT. It should trigger a complete noninvasive imaging protocol, including parenchymal and vascular MR imaging, to identify the underlying etiology. DSA should be performed secondarily only if the initial work-up remains inconclusive.

\section{Acknowledgments}

We thank David Seidenwurm, MD, for his help in editing this manuscript.

\section{References}

1. van Gijn J, Rinkel GJ. Subarachnoid haemorrhage: diagnosis, causes and management. Brain 2001;124(pt 2):249-78

2. Spitzer C, Mull M, Rohde V, et al. Non-traumatic cortical subarachnoid haemorrhage: diagnostic work-up and aetiological background. Neuroradiology 2005;47:525-31. Epub 2005 Jun 22
3. Patel KC, Finelli PF. Nonaneurysmal convexity subarachnoid hemorrhage. Neurocrit Care 2006;4:229-33

4. Refai D, Botros JA, Strom RG, et al. Spontaneous isolated convexity subarachnoid hemorrhage: presentation, radiological findings, differential diagnosis, and clinical course. J Neurosurg 2008;109:1034-41

5. Oppenheim C, Domigo V, Gauvrit JY, et al. Subarachnoid hemorrhage as the initial presentation of dural sinus thrombosis. AJNR Am J Neuroradiol 2005;26:614-17

6. Benabu Y, Mark L, Daniel S, et al. Cerebral venous thrombosis presenting with subarachnoid hemorrhage: case report and review. Am J Emerg Med 2009;27:96-106

7. Ducros A, Boukobza M, Porcher R, et al. The clinical and radiological spectrum of reversible cerebral vasoconstriction syndrome: a prospective series of 67 patients. Brain 2007;130:3091-101

8. Kumar R, Wijdicks EF, Brown RD Jr, et al. Isolated angiitis of the CNS presenting as subarachnoid haemorrhage. J Neurol Neurosurg Psychiatry 1997;62: $649-51$

9. Jain R, Deveikis J, Hickenbottom S, et al. Varicella-zoster vasculitis presenting with intracranial hemorrhage. AJNR Am J Neuroradiol 2003;24:971-74

10. Kannoth S, Iyer R, Thomas SV, et al. Intracranial infectious aneurysm: presentation, management and outcome. J Neurol Sci 2007;256:3-9

11. Osanai T, Kuroda S, Nakayama N, et al. Moyamoya disease presenting with subarachnoid hemorrhage localized over the frontal cortex: case report. Surg Neurol 2008;69:197-200

12. Shah AK. Non-aneurysmal primary subarachnoid hemorrhage in pregnancyinduced hypertension and eclampsia. Neurology 2003;61:117-20

13. Karabatsou K, Lecky BR, Rainov NG, et al. Cerebral amyloid angiopathy with symptomatic or occult subarachnoid haemorrhage. Eur Neurol 2007;57: $103-05$

14. Lieu AS, Howng SL. Intracranial meningioma with hemorrhage. Kaohsiung J Med Sci 1999;15:69-74

15. Vilela P, Saraiva P, Goulao A. Intracranial angiolipoma as cause of subarach- 
noid haemorrhage: case report and review of the literature. Neuroradiology 2005;47:91-96

16. Hentschel $S$, Toyota B. Intracranial malignant glioma presenting as subarachnoid hemorrhage. Can J Neurol Sci 2003;30:63-66

17. Rhode V, van Oosterhout A, Mull M, et al. Subarachnoid haemorrhage as initial symptom of multiple brain abscesses. Acta Neurochir (Wien) 2000;142: 205-08

18. Kleinig TJ, Kiley M, Thompson PD. Acute convexity subarachnoid haemorrhage: a cause of aura-like symptoms in the elderly. Cephalalgia 2008; 28:658-63. Epub 2008 Apr 16

19. Mitchell P, Wilkinson ID, Hoggard N, et al. Detection of subarachnoid haemorrhage with magnetic resonance imaging. J Neurol Neurosurg Psychiatry 2001; 70:205-11

20. Maeda M, Yagishita A, Yamamoto T, et al. Abnormal hyperintensity within the subarachnoid space evaluated by fluid-attenuated inversion-recovery MR imaging: a spectrum of central nervous system diseases. Eur Radiol 2003; 13(suppl 4):L192-201

21. Stuckey SL, Goh TD, Heffernan T, et al. Hyperintensity in the subarachnoid space on FLAIR MRI. AJR Am J Roentgenol 2007;189:913-21

22. Tha KK, Terae S, Kudo K, et al. Differential diagnosis of hyperintense cerebrospinal fluid on fluid-attenuated inversion recovery images of the brain. Part II. Non-pathological conditions. Br J Radiol 2009;82:610-14

23. da Rocha AJ, da Silva CJ, Gama HP, et al. Comparison of magnetic resonance imaging sequences with computed tomography to detect low-grade subarachnoid hemorrhage: role of fluid-attenuated inversion recovery sequence. J Comput Assist Tomogr 2006;30:295-303

24. Kamran S, Bener AB, Alper D, et al. Role of fluid-attenuated inversion recovery in the diagnosis of meningitis: comparison with contrast-enhanced magnetic resonance imaging. J Comput Assist Tomogr 2004;28:68-72

25. Singh SK, Leeds NE, Ginsberg LE. MR imaging of leptomeningeal metastases: comparison of three sequences. AJNR Am J Neuroradiol 2002;23:817-21

26. Villalobos-Chavez F, Rodriguez-Uranga JJ, Sanz-Fernandez G. Sequential changes in magnetic resonance in a limbic status epilepticus [in Spanish]. Rev Neurol 2005; 40:354-57

27. Frigon C, Jardine DS, Weinberger E, et al. Fraction of inspired oxygen in relation to cerebrospinal fluid hyperintensity on FLAIR MR imaging of the brain in children and young adults undergoing anesthesia. AJR Am J Roentgenol 2002;179:791-96

28. Filippi CG, Ulug AM, Lin D, et al. Hyperintense signal abnormality in subarachnoid spaces and basal cisterns on MR images of children anesthetized with propofol: new fluid-attenuated inversion recovery finding. AJNR Am J Neuroradiol 2001;22:394-99

29. Stoner T, Braff S, Khoshyomn S. High signal in subarachnoid spaces on FLAIR MR images in an adult with propofol sedation. Neurology 2002;59:292

30. Kim EY, Kim SS, Na DG, et al. Sulcal hyperintensity on fluid-attenuated inversion recovery imaging in acute ischemic stroke patients treated with intraarterial thrombolysis: iodinated contrast media as its possible cause and the association with hemorrhagic transformation. J Comput Assist Tomogr 2005; 29:264-69

31. Morris JM, Miller GM. Increased signal in the subarachnoid space on fluidattenuated inversion recovery imaging associated with the clearance dynamics of gadolinium chelate: a potential diagnostic pitfall. AJNR Am J Neuroradiol 2007;28:1964-67

32. Fellner FA, Fellner C, Aichner FT, et al. Importance of T2*-weighted gradientecho MRI for diagnosis of cortical vein thrombosis. Eur J Radiol 2005;56: 235-39

33. Idbaih A, Boukobza M, Crassard I, et al. MRI of clot in cerebral venous thrombosis: high diagnostic value of susceptibility-weighted images. Stroke 2006;37:991-95

34. Boukobza M, Crassard I, Bousser MG, et al. MR imaging features of isolated cortical vein thrombosis: diagnosis and follow-up. AJNR Am J Neuroradiol 2009;30:344-48

35. Haacke EM, Xu Y, Cheng YC, et al. Susceptibility weighted imaging (SWI). Magn Reson Med 2004;52:612-18

36. Linn J, Herms J, Dichgans M, et al. Subarachnoid hemosiderosis and superficial cortical hemosiderosis in cerebral amyloid angiopathy. AJNR Am J Neuroradiol 2008;29:184-86

37. Santhosh K, Kesavadas C, Thomas B, et al. Susceptibility weighted imaging: a new tool in magnetic resonance imaging of stroke. Clin Radiol 2009;64:74-83

38. Stam J. Thrombosis of the cerebral veins and sinuses. N Engl J Med 2005;352: 1791-98

39. Linn J, Ertl-Wagner B, Seelos KC, et al. Diagnostic value of multidetector-row
CT angiography in the evaluation of thrombosis of the cerebral venous sinuses. AJNR Am J Neuroradiol 2007;28:946-52

40. Leach JL, Fortuna RB, Jones BV, et al. Imaging of cerebral venous thrombosis: current techniques, spectrum of findings, and diagnostic pitfalls. Radiographics 2006;26(suppl 1):S19-41, discussion S42-43

41. Escott EJ, Rubinstein D, Cajade-Law AG, et al. Suprasellar cavernous malformation presenting with extensive subarachnoid hemorrhage. Neuroradiology 2001;43:313-16

42. Call GK, Fleming MC, Sealfon S, et al. Reversible cerebral segmental vasoconstriction. Stroke 1988;19:1159-70

43. Calabrese LH, Dodick DW, Schwedt TJ, et al. Narrative review: reversible cerebral vasoconstriction syndromes. Ann Intern Med 2007;146:34-44

44. Schwedt TJ, Matharu MS, Dodick DW. Thunderclap headache. Lancet Neurol 2006;5:621-31

45. Rossi CM, Di Comite G. The clinical spectrum of the neurological involvement in vasculitides. J Neurol Sci 2009;285:13-21

46. Chukwudelunzu FE, Brown RD Jr, Wijdicks EF, et al. Subarachnoid haemorrhage associated with infectious endocarditis: case report and literature review. Eur J Neurol 2002;9:423-27

47. Kim SJ, Lee JY, Kim TH, et al. Imaging of the neurological complications of infective endocarditis. Neuroradiology 1998;40:109-13

48. Krapf H, Skalej M, Voigt K. Subarachnoid hemorrhage due to septic embolic infarction in infective endocarditis. Cerebrovasc Dis 1999;9:182-84

49. Ake JA, Erickson JC, Lowry KJ. Cerebral aneurysmal arteriopathy associated with HIV infection in an adult. Clin Infect Dis 2006;43:e46-50

50. Jacobi C, Schwark C, Kress B, et al. Subarachnoid hemorrhage due to Borrelia burgdorferi-associated vasculitis. Eur J Neurol 2006;13:536-38

51. Weigel S, Kloska S, Kehl HG, et al. Lethal subarachnoid bleeding under immunosuppressive therapy due to mycotic arteritis. Eur Radiol 2003;13(suppl 6):L215-18

52. Marushima A, Yanaka K, Matsuki T, et al. Subarachnoid hemorrhage not due to ruptured aneurysm in Moyamoya disease. J Clin Neurosci 2006;13:146-49

53. Scott RM, Smith ER. Moyamoya disease and Moyamoya syndrome. N Engl J Med 2009;360:1226-37

54. Servillo G, Striano P, Striano S, et al. Posterior reversible encephalopathy syndrome (PRES) in critically ill obstetric patients. Intensive Care Med 2003;29: 2323-26

55. Bartynski WS. Posterior reversible encephalopathy syndrome. Part 2. Controversies surrounding pathophysiology of vasogenic edema. AJNR Am J Neuroradiol 2008;29:1043-49

56. Bartynski WS. Posterior reversible encephalopathy syndrome. Part 1. Fundamental imaging and clinical features. AJNR Am J Neuroradiol 2008;29: $1036-42$

57. Hefzy HM, Bartynski WS, Boardman JF, et al. Hemorrhage in posterior reversible encephalopathy syndrome: imaging and clinical features. AJNR Am J Neuroradiol 2009;30:1371-79. Epub 2009 Apr 22

58. Koch S, Rabinstein A, Falcone S, et al. Diffusion-weighted imaging shows cytotoxic and vasogenic edema in eclampsia. AJNR Am J Neuroradiol 2001;22: $1068-70$

59. McKinney AM, Short J, Truwit CL, et al. Posterior reversible encephalopathy syndrome: incidence of atypical regions of involvement and imaging findings. AJR Am J Roentgenol 2007;189:904-12

60. Doss-Esper CE, Singhal AB, Smith MS, et al. Reversible posterior leukoencephalopathy, cerebral vasoconstriction, and strokes after intravenous immune globulin therapy in Guillain-Barre syndrome. J Neuroimaging 2005;15:188-92

61. Knudsen KA, Rosand J, Karluk D, et al. Clinical diagnosis of cerebral amyloid angiopathy: validation of the Boston criteria. Neurology 2001;56:537-39

62. Ohshima T, Endo T, Nukui H, et al. Cerebral amyloid angiopathy as a cause of subarachnoid hemorrhage. Stroke 1990;21:480-83

63. Brunot S, Fromont A, Ricolfi F, et al. Focal subarachnoid hemorrhage and cerebral amyloid angiopathy: a non-fortuitous association [in French]. Rev Neurol (Paris) 2009 Mar 16. [Epub ahead of print]

64. Cuvinciuc V, Viguier A, Bonneville F. Cerebral amyloid angiopathy: a forgotten cause of sub-arachnoid hemorrhage [in French]. J Neuroradiol 2009;36: 245-46, author reply 246-47. Epub 2009 Sep 18

65. Katoh M, Yoshino M, Asaoka K, et al. A restricted subarachnoid hemorrhage in the cortical sulcus in cerebral amyloid angiopathy: could it be a warning sign? Surg Neurol 2007;68:457-60

66. Takeda S, Yamazaki K, Miyakawa T, et al. Subcortical hematoma caused by cerebral amyloid angiopathy: does the first evidence of hemorrhage occur in the subarachnoid space? Neuropathology 2003;23:254-61

67. Rogers LR. Cerebrovascular complications in cancer patients. Neurol Clin 2003;21:167-92 